\title{
Epigenetic changes during sepsis: on your marks!
}

\author{
Aurélien Bataille ${ }^{1,2^{*}}$, Pierre Galichon ${ }^{2,3}$, Marie-Julia Ziliotis ${ }^{2}$ Iman Sadia ${ }^{2}$ and Alexandre Hertig Im $^{2,3}$ \\ See related research by Bomsztyk et al. http://www.ccforum.com/content/19/1/225
}

\begin{abstract}
Epigenetics is the study of how cells, organs, and even individuals utilize their genes over specific periods of time, and under specific environmental constraints. Very importantly, epigenetics is now expanding into the field of medicine and hence should provide new information for the development of drugs. Bomsztyk and colleagues have detected major epigenetic changes occurring in several organs as early as $6 \mathrm{~h}$ after the onset of a mouse model of multiple organ dysfunction syndrome induced by Staphylococcus aureus lung injury. Decrease in mRNA of key genes involved in endothelial function was found to be associated with (and potentially explained by) a decrease in permissive histone marks, while repressive marks were unchanged. We discuss here the limitations of a whole-organ as opposed to a cell-specific approach, the nature of the controls that were chosen, and the pitfalls of histone modifications as a cause of the eventual phenotype. While the use of 'epidrugs' is definitely welcome in the clinic, how and when they will be used in sepsis-related multiple organ dysfunction will require further experimental studies.
\end{abstract}

\section{Commentary}

Progress in the science of epigenetics has increased our understanding of the interaction between living eukaryotes and the environment. We read with great interest the article by Karol Bomsztyk et al., recently published in Critical Care [1]. To our knowledge, this

\footnotetext{
* Correspondence: aurelbataille@gmail.com

${ }^{1}$ Department of Anesthesia and Intensive Care, Groupe Hospitalier Universitaire Saint-Louis-Lariboisière-Fernand-Widal, 75010 Paris, France ${ }^{2}$ Inserm UMR_S 1155, "Rare and common kidney diseases, matrix remodelling and tissue repair", Hôpital Tenon, 75020 Paris, France Full list of author information is available at the end of the article
}

is the first time that the community of intensivists has been provided with translational research exploring a major aspect of epigenetics (here, histone modifications) in sepsisrelated multiple organ dysfunction syndrome (MODS).

Epigenetics is a comparatively recent science, rapidly expanding in the field of medicine. Its definition has greatly evolved during the past century. At present it is defined as 'the structural adaptation of chromosomal regions so as to register, signal or perpetuate altered activity states' [2]. Physically, covalent modifications of histones (the proteins around which DNA is enwrapped, constituting nucleosomes) and methylation of DNA itself are two major biochemical changes strongly influencing how cells, organs, and even individuals make use of their genes over specific periods of time, and under specific environmental constraints. The reason for this is that some of these biochemical marks actually determine the accessibility of genes to RNA polymerase II and to relevant transcription factors. If we could identify if, when and how a given epigenetic mark, or a combination of marks, is induced by an injury, and also understand its real biological impact (good or bad) on the clinical outcome of that injury, then promoting or erasing these marks by using drugs - 'epidrugs' - could potentially represent a major breakthrough. By its severity, and the wide repercussions on a number of organs, sepsis exemplifies an event where epigenetics could help to reach unmet needs. Just like goal-directed resuscitation, based on pathophysiology and now a source of therapeutic targets [3], epigenetic modifications could very well be the future for care of patients with MODS: epidrugs are coming.

Endothelial cell dysfunction is a hallmark of sepsis. Bomsztyk et al. give insights into how epigenetic modifications are involved by using a mouse model of MODS induced by acute lung injury by Staphylococcus aureus. In short, they observed at the early time point of $6 \mathrm{~h}$ that the decrease in mRNA of key genes for endothelial function was associated with a decrease in histone marks known to be permissive (that is, to facilitate 
transcription). As a comparison, repressive marks were not induced at this time point. This suggests that an early intervention preserving or restoring these epigenetic marks would help to preserve endothelial integrity during MODS.

In our opinion, however, three issues need to be addressed to ensure the validity of this promising approach. The first one is technical but of utmost importance: which cell subtype should be studied when considering the epigenome? In homeostasis, many cells coexist in every organ, each with a similar genome but with its own epigenome: it is the very definition of epigenetics. In sepsis, there is a massive infiltration of immune cells in target organs, which makes a cell-specific approach (as opposed to an organ-specific one) even more essential. This is exemplified by the Neutrophil gelatinase-asssociated lipocalin (NGAL), a well-known marker of acute kidney injury, yet abundantly produced by neutrophils [4]. Here, NGAL is shown to be upregulated in the three organs studied, namely the kidney, the lung, and the liver, but with different epigenetic patterns: permissive acetylation of lysine 4 and 9 of histone $\mathrm{H} 3$ is increased in exon 1 in the liver and lung, but not in the kidney. Conversely, repressive methylation of lysine 27 of histone 3 decreases in the lung while increasing in the kidney. This emphasizes the need to integrate the different epigenetic marks in order to not only understand the activity of polymerase 2 in this region of the genome, but also to elucidate which cell acquires or loses these marks. Short of a cell-specific approach, results are complex and potentially confusing. One could speculate that the NGAL gene is differentially regulated in renal and inflammatory cells. We encountered similar problems and recently proposed ex vivo cell sorting to circumvent this issue in the kidney, at least in experimental conditions [5].

The second point refers to the control arm: mice undergo neither anesthesia nor mechanical ventilation. The authors argue that a previous microarray did not show substantial decrease in genes of interest, but this does not imply that the epigenome is stable. In addition, anesthesia itself - here isoflurane - has been shown to modify histone marks [6]. We would like to see evidence that, at least for some genes, polymerase 2 is active in endothelial cells; the authors only show negative marks (a decrease in permissive histone modifications, with a decrease in RNA polymerase II density). As explained above, choosing NGAL as a positive control takes the focus away from the endothelium and is still compatible with vascular rarefaction. A 'positive' control induced by MODS in endothelial cells is required [7-9].

Finally, how to articulate the cause of injury, the studied phenotype (here, endothelial dysfunction), and epigenetic marks is a general challenge in this emerging field of medicine. This necessitates analysis of the chronology and biological mechanisms whereby epigenetic marks are being bound. Cell metabolism, cell cross-talk, cytokines, and pathogen motifs may all be at issue. Bomsztyk and coworkers have made an important contribution by showing evidence of differences in RNA polymerase II activity. Upstream, cell-oriented mechanisms during sepsis must be clarified if we want to devise new therapies at given time points. We envisage that the impaired energy metabolism observed in sepsis [10] could drive many epigenetic changes. Knowing the highly dynamic nature of epigenetics, with some marks being furtive and others durable, timing of observation surely plays a critical role in how we interpret data; hence the need to be cautious about the causality of pathological changes that might only be temporary. The functional impact of one specific mark is still uncertain.

\section{Conclusion}

Epigenetic studies of systemic diseases such as sepsisrelated MODS may help to understand how cell damage proceeds. Obviously, experimental models are essential to explore causality. However, precision regarding the cell subtype involved in epigenetic changes is mandatory, and the timing of analysis is crucial to determine when epidrugs could be incremented in the clinic.

\section{Abbreviations}

MODS: Multiple organ dysfunction syndrome; NGAL: Neutrophil gelatinaseassociated lipocalin.

\section{Competing interests}

The authors declare that they have no competing interests.

\section{Authors' contributions}

$A B$ was responsible for the conception of the commentary. PG, MJZ, IS and $\mathrm{AH}$ helped to draft the manuscript. All authors read and approved the final manuscript.

\section{Authors' information}

All authors are part of the EPIK group (exploring EPIgenetic changes in acute Kidney injury).

\section{Acknowledgements}

The EPIK group is financially supported by a contract ( $A H$ and $A B$ ) with the French Institute of Health and Medical Research (Inserm), a contract (AB) with the Société Française d'Anesthésie-Réanimation (SFAR) and by the Foundation for Medical Research (FRM).

\section{Author details}

${ }^{1}$ Department of Anesthesia and Intensive Care, Groupe Hospitalier Universitaire Saint-Louis-Lariboisière-Fernand-Widal, 75010 Paris, France. 2Inserm UMR_S 1155, "Rare and common kidney diseases, matrix remodelling and tissue repair", Hôpital Tenon, 75020 Paris, France. ${ }^{3}$ Urgences Néphrologiques et Transplantation Rénale, Hôpital Tenon, 75020 Paris, France.

Published online: 15 October 2015

\section{References}

1. Bomsztyk K, Mar D, An D, Sharifian R, Mikula M, Gharib SA, et al. Experimental acute lung injury induces multi-organ epigenetic 
modifications in key angiogenic genes implicated in sepsis-associated endothelial dysfunction. Crit Care. 2015;19:225.

2. Bird A. Perceptions of epigenetics. Nature. 2007:447:396-8.

3. Rivers E, Nguyen B, Havstad S, Ressler J, Muzzin A, Knoblich B, et al. Early goal-directed therapy in the treatment of severe sepsis and septic shock. N Engl J Med. 2001;345:1368-77.

4. Mishra J, Ma Q, Prada A, Mitsnefes M, Zahedi K, Yang J, et al. Identification of neutrophil gelatinase-associated lipocalin as a novel early urinary biomarker for ischemic renal injury. J Am Soc Nephrol. 2003;14:2534-43.

5. Legouis D, Bataille A, Hertig A, Vandermeersch S, Simon N, Rondeau E, et al. Ex vivo analysis of renal proximal tubular cells. BMC Cell Biol. 2015;16:12.

6. Zhong T, Qing QJ, Yang Y, Zou WY, Ye Z, Yan JQ, et al. Repression of contexual fear memory induced by isoflurane is accompanied by reduction in histone acetylation and rescued by sodium butyrate. Br J Anaesth. 2014;113:634-43.

7. Joyce DE, Gelbert L, Ciaccia A, DeHoff B, Grinnell BW. Gene expression profile of antithrombotic protein $\mathrm{C}$ defines new mechanisms modulating inflammation and apoptosis. J Biol Chem. 2001;276:11199-203.

8. Ostrowski SR, Haase N, Müller RB, Møller MH, Pott FC, Perner A, et al. Association between biomarkers of endothelial injury and hypocoagulability in patients with severe sepsis: a prospective study. Crit Care. 2015;19:191.

9. Skibsted S, Jones AE, Puskarich MA, Arnold R, Sherwin R, Trzeciak S, et al. Biomarkers of endothelial cell activation in early sepsis. Shock. 2013;39:427-32.

10. Fink MP. Bench-to-bedside review: Cytopathic hypoxia. Crit Care. 2002;6:491-9. 